\title{
Efeito de técnicas de enriquecimento ambiental no comportamento de Leontopithecus chrysomelas (Kuhl, 1820) (Primates: Callitrichidae)
}

\author{
Denise Costa Rebouças Lauton ${ }^{1} \&$ Antônio de Oliveira Costa Neto ${ }^{2}$
}

(1) Universidade Federal do Pará, Instituto de Ciências Biológicas, Museu Paraense Emílio Goeldi, Programa de Pós-Graduação em Zoologia, Rua Augusto Corrêa 01, Guamá 66075-110, Belém, Pará, Brasil. E-mail: deniselauton@yahoo.com.br

(2) Universidade Estadual de Feira de Santana, Departamento de Ciências Biológicas, Avenida Transnordestina, Novo Horizonte 44036-900, Feira de Santana, Bahia, Brasil. E-mail: aocneto@uefs.br

Lauton D.C.R. \& Costa Neto A.O. (2018) Efeito de técnicas de enriquecimento ambiental no comportamento de Leontopithecus chrysomelas (Kuhl, 1820) (Primates: Callitrichidae). Pesquisa e Ensino em Ciências Exatas e da Natureza, 2(2): 86-94. http://dx.doi.org/10.29215/pecen.v2i2.1063

Editor acadêmico: Manoel dos Santos Filho. Recebido: 15 Março 2018. Aceito: 27 Junho 2018. Publicado: 28 Dezembro 2018.

Resumo: Enriquecimento ambiental consiste em técnicas que modificam o ambiente cativo, de forma a dinamizá-lo. A adoção do método resulta, com frequência, na diminuição de comportamentos anormais (estereotipias) e na expressão de um repertório comportamental mais típico. Acreditando-se na eficácia das técnicas de enriquecimento ambiental e que é possível melhorar a condição de indivíduos cativos, este trabalho foi proposto. Técnicas de enriquecimento Físico e Alimentar foram aplicadas a grupos e indivíduos solitários de Leontopithecus chrysomelas (Kuhl, 1820) mantidos no Laboratório Tropical de Primatologia (LTP), da Universidade Federal da Paraíba (UFPB). As observações foram realizadas em três fases: Controle; enriquecimento Físico; e enriquecimento Alimentar. $\mathrm{Na}$ fase Controle os indivíduos apresentaram comportamentos anormais frequentes, como o pacing e o vigiar excessivo, principalmente os indivíduos solitários. Durante a fase experimental, apenas o ato vigiar apresentou diferença significativa para os grupos familiares $\left(\mathrm{Fr}^{2}=7.58 ; \mathrm{gl}=2 ; \mathrm{p}=0.023\right)$. Os resultados demostram que $L$. chrysomelas do LTP parecem ser mais resistentes às mudanças, possivelmente devido ao longo período de exposição destes a um ambiente condicionado a uma rotina e sem estímulos. Visando modificar este cenário, sugere-se a adoção de métodos de "quebra de rotina" concomitante às técnicas de enriquecimento ambiental.

Palavras chave: Cativeiro, comportamento, Primata.

Effect of environmental enrichment techniques on the behavior of Leontopithecus chrysomelas (Kuhl, 1820) (Primates: Callitrichidae)

Abstract: Environmental enrichment consists in techniques that modify the captive environment, in order to make it more dynamic. The adoption of the method, often, results in the reduction of abnormal behavior (stereotypies) and expression of a more typical behavioral repertoire. Believing in the effectiveness of environmental enrichment techniques and that it is possible to improve the condition of captives individuals, this work was proposed. There were applied techniques of Physical and Feed enrichment to groups and solitary individuals captives of Leontopithecus chrysomelas (Kuhl, 1820) kept in Tropical Laboratory of Primatology (TLP), of the Universidade Federal da Paraíba (UFPB). The observations were made in three phases: Control; Enrichment of the Physical; and Nutritional. Individuals presented frequent abnormal behaviors during Control phase, such as pacing and excessive watching, especially the lonely individuals. During the experimental phase, only the act to guard presented significant difference for the family groups $\left(\mathrm{Fr}^{2}=7.58 ; \mathrm{gl}=2 ; \mathrm{p}=0.023\right)$. The results show that $L$. chrysomelas of the TLP seem to be more resistant to change, possibly due to the long period of exposition of these to a conditioned environment to a routine and without stimuli. Aimed to modify this scenario, it is suggested to adopt methods of "routine break" concomitant to the techniques of environmental enrichment.

Key words: Behavior, captivity, Primates. 


\section{Introdução}

Circunstâncias impostas pelo ambiente cativo, como a disponibilidade do recurso alimentar em horário pré-estabelecido, restrição física de espaço, falta de estímulos, dentre outras, propiciam que muitos dos animais que vivem sob esta situação apresentem comportamentos indicadores de estresse, distúrbios comportamentais, tais como as estereotipias, automutilação e depressão, e fisiológicos, como a obesidade ou a deficiência nutricional (Boere 2001; Box 2007; Buchanan-Smith 2010). Estereotipias parecem estar restritas a animais em ambiente cativo, são comportamentos caracterizados pela expressão de movimento invariável e repetitivo desprovido de propósito e função aparente (Boere 2001; Box 2007). Estereotipias locomotoras, como o pacing excessivo, posturas estereotipadas, como o balançar frequente da cabeça, dentre outras, são ações observadas frequentemente em primatas cativos e indicam fortes componentes aversivos ao ambiente no qual o animal vive (Buchanan-Smith 2010).

Sob tais condições impostas pelo ambiente cativo, técnicas de enriquecimento ambiental têm sido frequentemente empregadas, uma vez que procuram instigar e entreter o animal minimizando, com frequência, o desenvolvimento de comportamentos anormais (Carlstead \& Shepherdson 1994; Boere 2001; Pizzutto et al. 2009). As práticas de enriquecimento ambiental consistem na modificação do espaço físico do ambiente cativo e/ou na inserção de elementos que criem oportunidades de privacidade, de exploração e de convivência social (Carlstead \& Shepherdson 1994; Buchanan-Smith 2010). Esta dinâmica implementada possibilita aumento da expectativa e qualidade de vida dos indivíduos cativos, além de favorecer o sucesso reprodutivo dos mesmos (Carlstead \& Shepherdson 1994; Boere 2001; Pizzutto et al. 2009; Borges et al. 2011). Para facilitar a implantação das técnicas em recintos de primatas, Bloomsmith et al. (1991) as classificaram em cinco principais tipos: alimentar, social, sensorial, cognitiva e do ambiente físico. Cada uma destas categorias de enriquecimento pode interferir, em intensidade variável, no padrão de atividades dos indivíduos cativos, sendo necessário o conhecimento prévio das necessidades comportamentais da espécie para que sua adoção tenha resultados satisfatórios (Bloomsmith et al. 1991; Boere 2001; Buchanan-Smith 2010; Borges et al. 2011).

Indivíduos de Leontopithecus chrysomelas (Kuhl, 1820) são mantidos em recintos considerados empobrecidos no Laboratório Tropical de Primatologia - LTP da UFPB e apresentam distúrbios comportamentais, possivelmente como resposta a esta condição. Técnicas de enriquecimento Físico e Alimentar foram aplicadas a grupos e indivíduos solitários cativos de L. chrysomelas com objetivo de averiguar a eficácia do método e se, após esta exposição, possíveis problemas associados à vida em ambiente cativo, como a redução na frequência de comportamentos anormais e/ou exacerbados, foram amenizados. Para tanto comparamos a frequência dos registros comportamentais antes (fase controle) e durante a exposição aos enriquecimentos Físico e Alimentar.

\section{Material e Métodos}

\section{Local e grupo de estudo}

Este trabalho foi realizado durante os meses de agosto e setembro de 2010 no Laboratório Tropical de Primatologia (LTP) localizado na Universidade Federal da Paraíba Campus I, João Pessoa, Paraíba, Brasil. O LTP conta com um plantel de 36 indivíduos distribuídos em 7 espécies de primatas: Aotus nigriceps (Dollman, 1909), Callithrix jacchus (Linnaeus, 1758), Callithrix penicillata (É. Geoffroy, 1812), Callithrix geoffroyi (Humboldt, 1812), Sapajus nigritus (Linnaeus, 1758), Leontopithecus chrysomelas e o Saguinus midas (Linnaeus, 1758). Destes observamos dez indivíduos cativos de L. chrysomelas, sendo quatro mantidos em recintos de forma isolada (três fêmeas e um macho) e seis formando grupos familiares com três indivíduos cada (recinto 10: duas fêmeas e um macho; recinto 26: um macho adulto, um macho juvenil e uma fêmea). Diante da mudança de gestão e avanços tecnológicos, dados individuais dos espécimes sobre a sua origem e data de incorporação no plantel do LTP são imprecisos. No entanto, sabe-se que estes indivíduos de L. chrysomelas são cativos desde a 
implementação do LTP até então, a pouco mais de 10 anos. Os indivíduos dos grupos familiares foram distinguidos entre si por características sexuais e etárias, coloração do pelo e tamanho da cauda.

\section{Descrição dos recintos}

Os recintos dos indivíduos solitários apresentam dimensões de $1.5 \mathrm{~m}$ de largura x $1.5 \mathrm{~m}$ de comprimento x $1.8 \mathrm{~m}$ de altura (recinto 43, 41, 04 e 31); enquanto os recintos com composição familiar apresentam dimensões de 3.35 m largura $\times 3.35 \mathrm{~m}$ comprimento $\times 3 \mathrm{~m}$ de altura (recinto 10) e $1.60 \mathrm{~m}$ largura x $1.60 \mathrm{~m}$ comprimento x $1.75 \mathrm{~m}$ largura de altura (recinto 26). A constituição superior e lateral dos recintos é de tela losangular galvanizada com base para sustentação de tijolos. Os recintos apresentam lateralmente, um abrigo e uma plataforma de madeira sobre a qual se tem um recipiente plástico para água e outro para alimento. No espaço central do recinto há troncos de árvores que possibilitam o acesso rápido dos indivíduos às laterais opostas deste. A alimentação é ofertada aos animais duas vezes ao dia sempre no mesmo comedouro e em horários pré-estabelecidos. A primeira refeição era servida as 7:30 h e era composta por diversas frutas como banana, melancia, uva, abacaxi dentre outras. A segunda refeição era servida entre 11:30 e 13:00 h sendo composta por banana batida no liquidificador com leite, açúcar, vitamina-C e pão, alternando alguns dias esta dieta com pedaços de carne bovina e alface.

\section{Coleta dos dados}

O estudo foi realizado em três fases (Controle, Enriquecimento Físico e Enriquecimento Alimentar) precedidas por um período de observação preliminar, no qual, elaborou-se uma planilha de observação utilizada nas fases posteriores (Tabela 1) e, concomitantemente, acostumou-se os indivíduos cativos à presença do observador. Na primeira fase, denominada Controle, o comportamento habitual dos animais foi registrado. Posterior a esta, realizou-se o enriquecimento do ambiente físico (Figura 1). Para tanto, confeccionamos artefatos de tubos de PVC contendo aberturas que foram colocados na área superior dos recintos 04, 43 e 26 simulando troncos de árvores. Nos outros três recintos, 31, 41 e 10, colocou-se na grade lateral uma hélice de arame galvanizado contendo tampinhas de garrafa PET de diversas cores, como vermelho, azul, verde, dentre outras. A escolha do tipo de artefato para cada recinto foi aleatória através de sorteio. Na fase de enriquecimento Alimentar foram utilizados tubos de PVC contendo no seu interior larvas de Tenebrio molitor (Ordem Coleoptera) e, para manutenção, farelo de trigo e serrapilheira. Na superfície deste tubo haviam aberturas que possibilitavam o acesso dos indivíduos ao item alimentar. $\mathrm{O}$ artefato foi parcialmente forrado com sisal, para simular objetos de forrageio encontrados em seu ambiente natural, com grau de dificuldade para obtenção do recurso alimentar ativo (Figura 2).

Para observação dos indivíduos, foi utilizado o método animal-focal com registro instantâneo a cada um minuto (Altmann 1974), sendo de 10 minutos a duração de cada unidade amostral. As observações foram diárias, ocorrendo duas vezes por indivíduo, sendo realizadas nos dois períodos do dia (matutino e vespertino) na tentativa de reduzir possíveis influências diárias no comportamento dos indivíduos. Estas observações foram realizadas sempre depois de transcorridos mais de 30 minutos dos horários de desjejum e sem chuva, para que estes fatores não interferissem nas análises. No geral, foram 2400 registros de atividades, sendo 80 registros por indivíduo em cada fase.

\section{Análise dos dados}

O orçamento das atividades foi calculado pela frequência das ocorrências de cada categoria comportamental e expresso em porcentagens de registros. $\mathrm{O}$ Teste Friedman foi utilizado para verificar variações entre as frequências comportamentais observadas durante a fase Controle e as observadas durante as fases de enriquecimento. Para verificar diferenças na 
utilização dos artefatos entre as fases foi utilizado o teste de Wilcoxon. Estas análises foram realizadas para as diferentes composições de Leontopithecus chrysomelas (solitário x grupo), utilizando-se o software BIOESTAT 5.0 (Ayres et al. 2007) com nível de significância de p $\leq 0.05$.

Tabela 1. Etograma de Leontopithecus chrysomelas do Laboratório Tropical de Primatologia elaborado na fase preliminar deste estudo.

\begin{tabular}{|c|c|}
\hline Atos comportamentais & Descrição \\
\hline Autocatação ou "Grooming" & $\begin{array}{l}\text { Inspeção pelo indivíduo do seu próprio pelo com as mãos, dentes ou } \\
\text { língua para retirar parasitas ou sujeira. }\end{array}$ \\
\hline Catação Social ou "Grooming" & $\begin{array}{l}\text { Inspeção entre indivíduos. Eles se alternam na remoção de parasitas e } \\
\text { sujeiras dos pelos utilizando as mãos, dentes ou língua. }\end{array}$ \\
\hline Brincar & $\begin{array}{l}\text { Em indivíduos solitários este comportamento é verificado quando estes } \\
\text { rolam sobre si mesmo ou sobre a vasilha, manipulando-a. Já nos grupos } \\
\text { familiares, um indivíduo persegue ao outro com mordidas e/ou puxões } \\
\text { durante a ação. }\end{array}$ \\
\hline Vigiar & $\begin{array}{l}\text { Postura ereta do corpo e olhar atento à movimentação ao seu redor. O } \\
\text { indivíduo pode estar em locomoção. }\end{array}$ \\
\hline Descansar & $\begin{array}{l}\text { Sem atividade dinâmica aparente; embora possa estar observando o } \\
\text { ambiente, o indivíduo não se desloca do ponto onde se encontra. O } \\
\text { indivíduo pode estar em repouso temporário ou dormindo. }\end{array}$ \\
\hline Forragear & $\begin{array}{l}\text { Procura manual ou visual do alimento. Esta busca, com ou sem manuseio, } \\
\text { pode ocorrer em substratos com orifícios, cavidades ou fendas. }\end{array}$ \\
\hline Marcar & $\begin{array}{l}\text { Marcar, roçar o corpo nos galhos ou grades do recinto através das áreas } \\
\text { glandulares. }\end{array}$ \\
\hline Estereotipia - pacing & $\begin{array}{l}\text { Percorrer o recinto sempre na mesma trajetória de forma repetitiva e } \\
\text { prolongada, em alta frequência, geralmente de forma cíclica, sem } \\
\text { demonstrar uma finalidade específica. }\end{array}$ \\
\hline Manuseio com os artefatos & Interação com o artefato posto no recinto durante o enriquecimento I e II. \\
\hline Outros & $\begin{array}{l}\text { Atividades não contidas dentre as categorias anteriores, como beber água, } \\
\text { urinar, defecar e locomover-se. }\end{array}$ \\
\hline
\end{tabular}
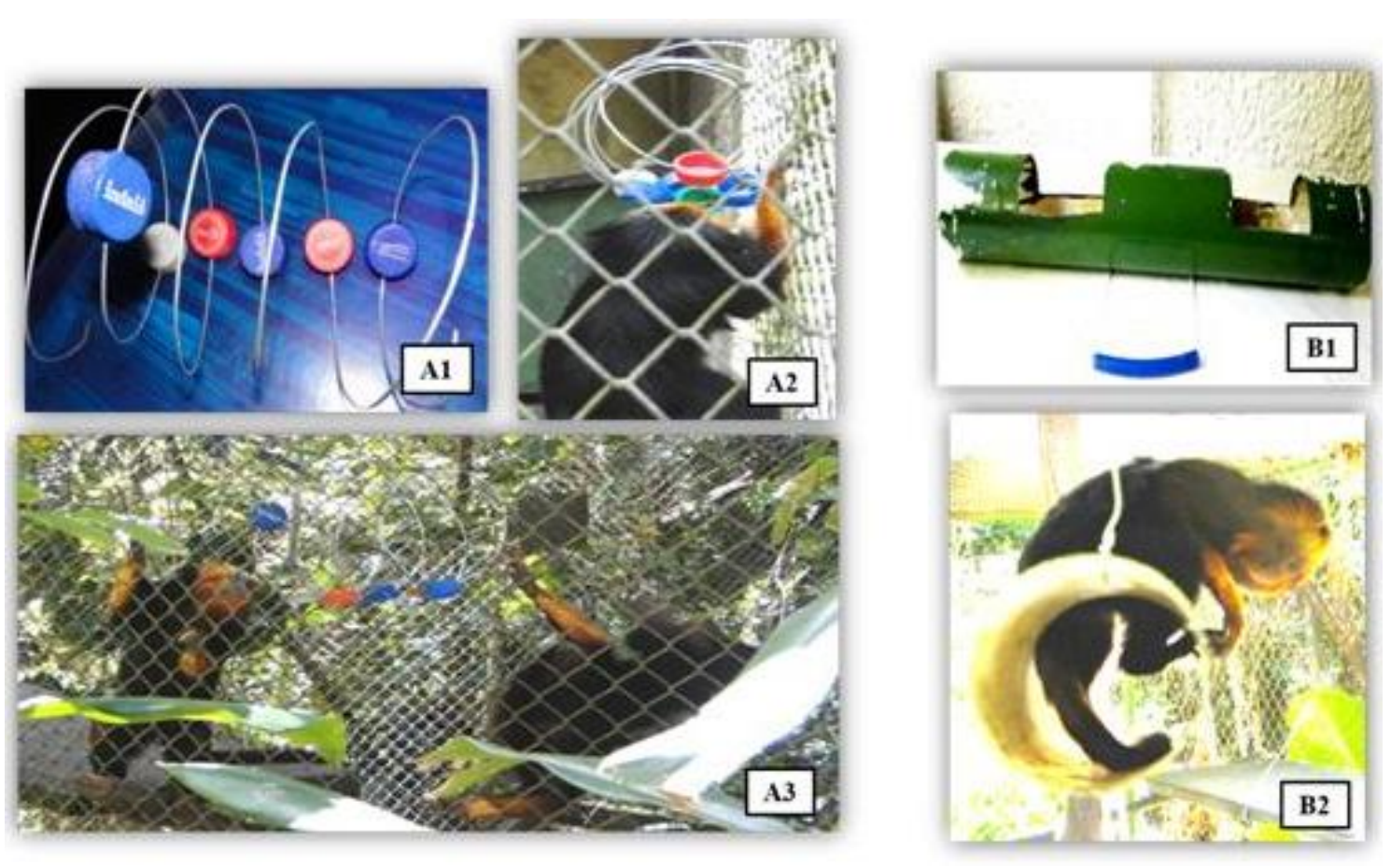

Figura 1. Enriquecimento do ambiente Físico para Leontopithecus chrysomelas no Laboratório Tropical de Primatologia: A1 - Artefato hélice de arame de ferro galvanizado com tampinhas de garrafa PET (32 cm de comprimento); A2 - Interação do indivíduo solitário com o artefato hélice; A3 - Interação dos grupos familiares com o artefato hélice; B1 - Tubo de PVC de $60 \mathrm{~cm}$ de comprimento com aberturas de $10 \mathrm{~cm}$ de comprimento; B2 - Artefato de PVC sendo manipulado. 

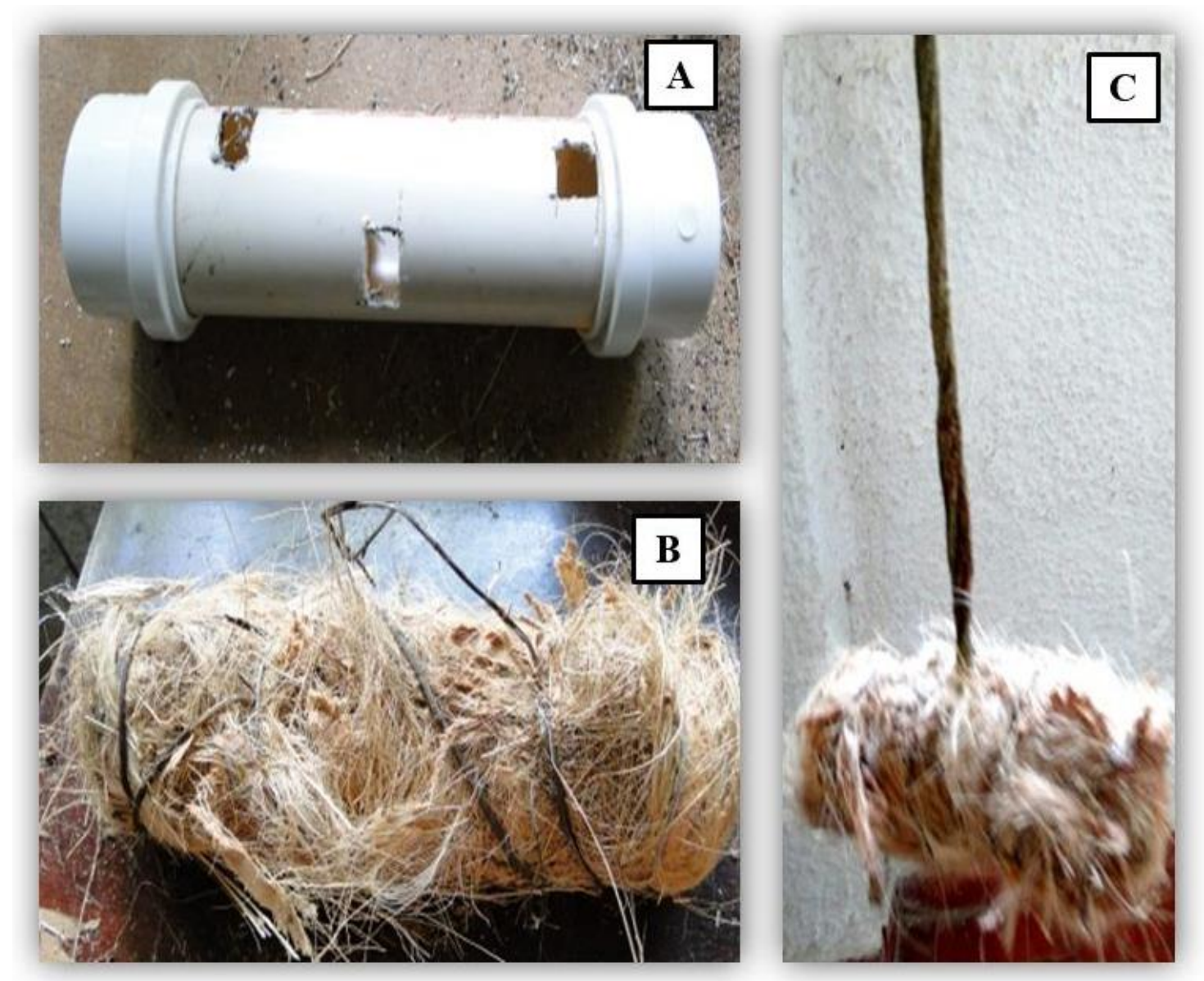

Figura 2. Enriquecimento Alimentar para Leontopithecus chrysomelas no Laboratório Tropical de Primatologia: A - Tubo de PVC (5 cm de diâmetro com aberturas de 4 a $6 \mathrm{~cm}$ ); B - Tubo de PVC com larvas de tenébrios no seu interior, forrado com sisal (Agave sisalana); C - Artefato PVC finalizado.

\section{Resultados}

Para indivíduos solitários de Leontopithecus chrysomelas, o orçamento geral de atividades durante a fase Controle caracterizou-se por uma maior proporção de tempo gasto nas categorias comportamentais vigiar $(43 \%)$, outros $(24 \%)$, descanso $(10 \%)$, forrageio $(9 \%)$, estereotipias $(8.4 \%)$, seguida por marcação $(3 \%)$, brincadeira (1.6\%) e auto-catação (1\%). Quando comparamos o orçamento de atividades apresentado durante a fase Controle ao apresentado durante as fases de enriquecimento Físico e Alimentar, não encontramos diferença significativa (Tabela 2).

Dos quatros indivíduos solitários observados, todos apresentaram estereotipias do tipo pacing na fase Controle (Figura 3). Após a utilização dos enriquecimentos, apesar da frequência alterar, diferenças significativas na expressão das estereotipias não foram observadas. Diferença significativa também não foi observada quanto à manipulação dos artefatos expostos durantes as fases de enriquecimento $(\mathrm{W}=2 ; \mathrm{gl}=1 ; \mathrm{p}=0.65)$.

Para os grupos cativos de Leontopithecus chrysomelas, o orçamento geral de atividades durante a fase Controle caracterizou-se por uma maior proporção de tempo gasto no ato vigiar $(30.6 \%)$, outros $(22.31 \%)$, forrageio $(18.54 \%)$, brincadeira $(10.4 \%)$, descanso $(10 \%)$, e, em menor frequência, pelos atos de catação social (3.5\%), marcação (1.7\%), auto-catação (1.7\%) e estereotipias $(1.25 \%)$. Variação do orçamento de atividades entre as fases foi verificada apenas para $\mathrm{o}$ ato vigiar tendo esta atividade maior frequência durante a fase de enriquecimento Físico. Para os outros atos não encontramos diferenças significativas entre as fases (Tabela 2).

Apenas um dos seis indivíduos dos grupos apresentou estereotipias na fase Controle, contudo em uma baixa frequência $(1.3 \% ; \mathrm{N}=6)$ (Figura 3). Dentre as duas técnicas adotadas 
para o enriquecimento dos recintos, verificou-se elevada frequência de manuseio ao enriquecimento Físico, entretanto não houve diferença significativa entre as duas técnicas de enriquecimento adotadas $(\mathrm{W}=4.5 ; \mathrm{gl}=1 ; \mathrm{p}=0.41)$. No geral, o macho juvenil do recinto 26 (MII) foi o que apresentou maior interação com os artefatos apresentados (e.g., enriquecimento I: $71 \%)$.

Tabela 2. Média e desvio padrão dos atos comportamentais expressos por grupos e indivíduos solitários de Leontopithecus chrysomelas durante as fases Controle, Enriquecimento Físico (EAI) e Enriquecimento Alimentar (EAII). Letras iguais na mesma linha demonstram que as médias não diferiram significativamente pelo teste Friedman.

\begin{tabular}{|c|c|c|c|c|c|c|}
\hline \multicolumn{7}{|c|}{ Composição } \\
\hline & \multicolumn{3}{|c|}{ Solitário } & \multicolumn{3}{|c|}{ Grupo } \\
\hline $\begin{array}{c}\text { Atos } \\
\text { comportamentais }\end{array}$ & Controle & EAI & EAII & Controle & EAI & EAII \\
\hline Vigiar & $34.5 \pm 3.11 \mathrm{~A}$ & $29.5 \pm 8.2 \mathrm{~A}$ & $37 \pm 5.94 \mathrm{~A}$ & $24.5 \pm 10.27 \mathrm{~A}$ & $30.16 \pm 8.6 \mathrm{~B}^{*}$ & $30.83 \pm 9.58 \mathrm{~B}$ \\
\hline Brincar & $1.25 \pm 1.26 \mathrm{~A}$ & $2.25 \pm 2.06 \mathrm{~A}$ & $0 \mathrm{~A}$ & $8.33 \pm 4.6 \mathrm{~A}$ & $6.66 \pm 3.07 \mathrm{~A}$ & $10 \pm 7.7 \mathrm{~A}$ \\
\hline Descansar & $8 \pm 7.26 \mathrm{~A}$ & $7.25 \pm 7.4 \mathrm{~A}$ & $5.5 \pm 6.45 \mathrm{~A}$ & $7.83 \pm 4.6 \mathrm{~A}$ & $6.33 \pm 2.94 \mathrm{~A}$ & $11 \pm 3.40 \mathrm{~A}$ \\
\hline Marcar & $2.25 \pm 0.5 \mathrm{~A}$ & $2 \pm 1.41 \mathrm{~A}$ & $1 \pm 0.82 \mathrm{~A}$ & $1.33 \pm 1.97 \mathrm{~A}$ & $2.33 \pm 1.51 \mathrm{~A}$ & $0.7 \pm 1.03 \mathrm{~A}$ \\
\hline Autocatação & $0.75 \pm 0.96 \mathrm{~A}$ & $1 \pm 1.15 \mathrm{~A}$ & $0.5 \pm 1 \mathrm{~A}$ & $1.33 \pm 1.36 \mathrm{~A}$ & $0 \mathrm{~A}$ & $0.17 \pm 0.41 \mathrm{~A}$ \\
\hline Estereotipia & $6.75 \pm 3.4 \mathrm{~A}$ & $5.25 \pm 9.85 \mathrm{~A}$ & $5 \pm 8.72 \mathrm{~A}$ & $1 \pm 2.45 \mathrm{~A}$ & $1.33 \pm 2.42 \mathrm{~A}$ & $1.83 \pm 2.99 \mathrm{~A}$ \\
\hline Forragear & $7.25 \pm 5.19 \mathrm{~A}$ & $12 \pm 9.97 \mathrm{~A}$ & $14.25 \pm 12.99 \mathrm{~A}$ & $14.83 \pm 8.23 \mathrm{~A}$ & $12.67 \pm 3.50 \mathrm{~A}$ & $11.5 \pm 7.09 \mathrm{~A}$ \\
\hline Catação Social & - & --- & -- & $2.83 \pm 1.94 \mathrm{~A}$ & $1.83 \pm 2.64 \mathrm{~A}$ & $1.67 \pm 1.4 \mathrm{~A}$ \\
\hline
\end{tabular}

Diferença entre a fase Controle e de EAI do ato vigiar: $\mathrm{Fr}^{2}=7.58 ; \mathrm{gl}=2 ; \mathrm{p}=0.023$

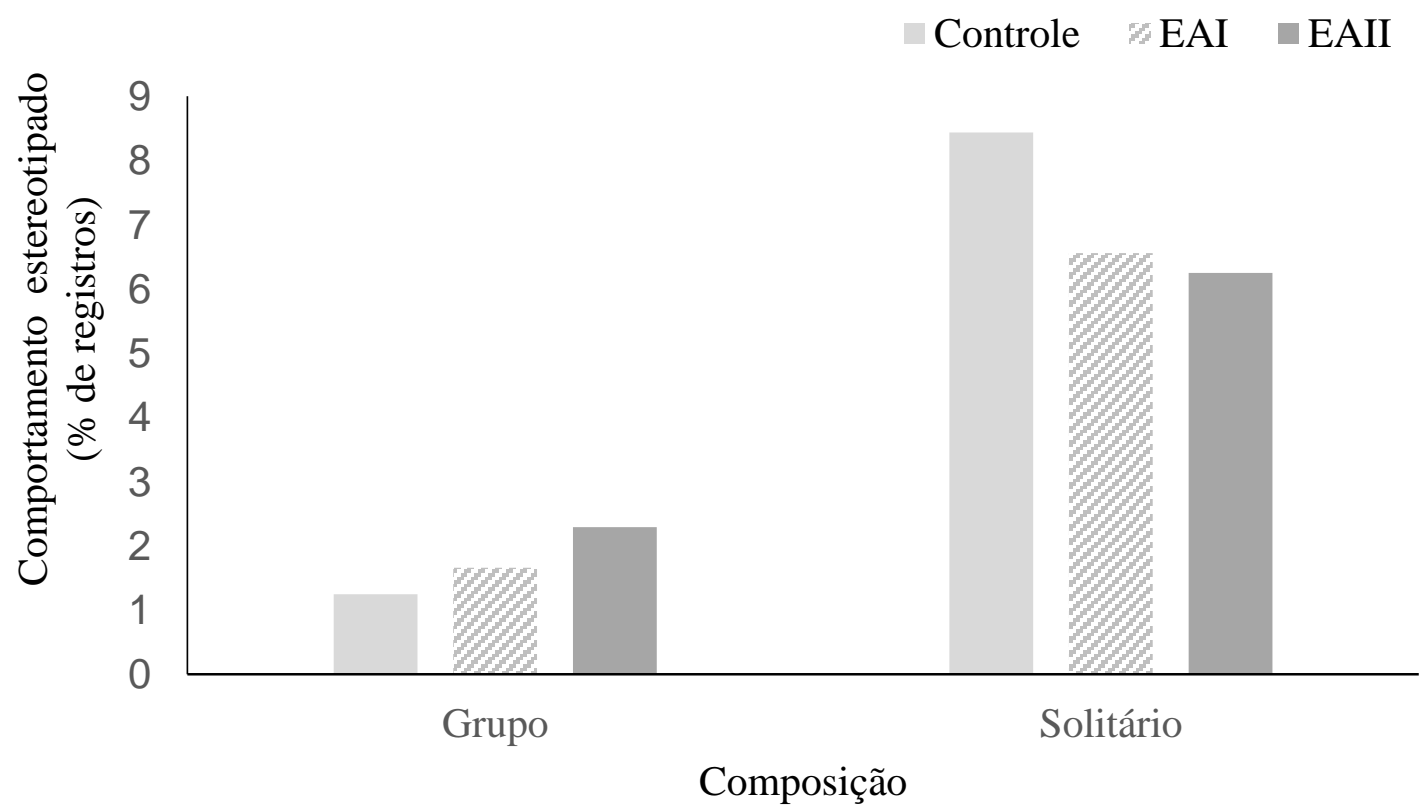

Figura 3. Comportamento estereotipado expresso por grupos e indivíduos solitários de Leontopithecus chrysomelas nas fases Controle, Enriquecimento Físico (EAI) e Enriquecimento Alimentar (EAII).

\section{Discussão}

Os indivíduos de Leontopithecus chrysomelas mantidos no Laboratório Tropical de Primatologia encontram-se em um ambiente reduzido, sem estímulos motores, privados da convivência social, com fácil acesso à comida e desjejuns em horários fixos. Tais condições caracterizam o ambiente cativo como um meio condicionado a uma rotina e sem estímulos, fatores que, aliado ao longo período cativo, contribuíram para o desenvolvimento de comportamentos anormais verificados neste estudo.

Leontopithecus chrysomelas em vida livre despende a maior parte do tempo à locomoção, ao forrageio e à alimentação (Raboy \& Dietz 2004; Guidorizzi 2008; Reis 2012). O ato 
vigiar, diferente do observado em $L$. chrysomelas de vida livre, se destaca neste estudo, tanto para os indivíduos solitários quanto para os de composição familiar, representando $1 / 2$ da frequência das atividades de L. chrysomelas cativos do LTP. Esta expressão exacerbada do ato vigiar possivelmente indica ansiedade ou medo antecipado proporcionado pelo ambiente cativo (Boere 2001). Os visitantes recebidos pelo LTP parecem influenciar nesta postura, deixando-os mais agitados e ariscos. Chamove et al. (1988) já haviam destacado o efeito negativo de visitantes sobre os primatas. A variação significativa do ato vigiar nos grupos familiares, em média seis registros a mais que na fase controle $\left(\mathrm{Fr}^{2}=7.58\right.$; $\left.\mathrm{gl}=2 ; \mathrm{p}=0.023\right)$, coincide com o início da entrada de grupos de visitantes ao LTP, acontecimento que pode justificar a variação na expressão deste comportamento. Entretanto, mais observações devem ser realizadas com objetivo de compreender melhor o fato.

Nossos dados demonstram que a expressão de comportamentos estereotipados é frequente nos indivíduos solitários quando comparado aos indivíduos dos grupos familiares. Este acontecimento possivelmente provém da necessidade básica de calitriquídeos ao convívio social. Quando na ausência deste convívio, os calitriquídeos parecem recorrer a outras atividades, muitas vezes sem objetivo específico, como a expressão de estereotipias, conforme já observado por Borges et al. (2011) e Castillioni \& Andrade (2009) em calitriquídeos cativos. Reis (2012), ao estudar grupos de Leontopithecus chrysomelas em vida livre (área de cabruca) verificou que estes investiam grande parte do tempo em atividades sociais (40\%). Segundo Boere (2001), este gregarismo observado em primatas, propicia o enfretamento de situações estressantes e a expressão de comportamentos naturais e típicos da espécie, sendo desejável e enriquecedor a formação de agrupamentos em ambientes cativos para proporcionar melhores condições ao animal. Ao estudar L. chrysomelas cativos, Gregório \& Andrade (2010) verificaram que, quando em composição familiar, estes apresentam repertório comportamental variado e ausência de comportamentos atípicos.

Ao introduzir técnicas de enriquecimento Físico e Alimentar a Leontopithecus chrysopygus (Mikan, 1823) cativos, Castillioni \& Andrade (2009) observaram a redução na frequência de comportamentos atípicos e estereotipados, possivelmente um indício de diminuição do estresse ocasionado pelo ambiente cativo (Almeida et al. 2008; Borges et al. 2011). Entretanto, neste estudo, esta variação não foi verificada. Aparentemente houve uma perda rápida do interesse de $L$. chrysomelas aos artefatos apresentados e estes não se estabeleceram, apesar dos artefatos elaborados neste trabalho e duração de exposição serem similares aos indicados pelas normas de enriquecimento propostas pela European Association of Zoos and Aquaria (EAZA) para calitriquídeos (Ruivo 2010). Supõe-se, portanto, que esse resultado pode estar sendo influenciado pelo fato de que os indivíduos de $L$. chrysomelas mantidos no LTP estão a um longo período expostos a um ambiente condicionado a uma rotina e sem estímulos sendo, logo, mais inflexíveis às mudanças.

Como proposto por Boere (2001), a biologia da espécie foi considerada na elaboração dos enriquecimentos e por isto esperava-se uma maior exploração dos artefatos apresentados. Dentre os artefatos, contava-se com uma maior exploração do artefato que continha alimentos (fase de Enriquecimento Alimentar), uma vez que este oferecia um recurso vivo, larvas de tenébrio, da ordem Coleoptera, que segundo Kierulff et al. (2008), é a mais consumida por Leontopithecus chrysomelas em seu ambiente natural. Embora a frequência de manipulação do artefato tenha sido baixa, pode-se verificar que houve interação do indivíduo com o artefato proposto, pois este era encontrado revirado em período anterior ao início das observações diárias.

De forma geral, uma variação significativa no padrão comportamental da espécie não foi observada com os enriquecimentos. Este resultado demonstra a necessidade de uma verificação, com estes indivíduos, de outras técnicas de enriquecimento ambiental que surtam efeito a longo prazo aliada a "quebra alimentar" descrita por Borges et al. (2011) (variações/quebra na rotina dos horários de oferta, dos locais e dos recipientes de alimentação) para que o efeito do longo período expostos dos indivíduos ao confinamento e a rotina seja minimizado e que as técnicas sejam mais efetivas. Estas, dentre outras interferências, podem 
gerar resultados positivos em trabalhos de enriquecimento ambiental com Leontopithecus chrysomelas.

\section{Agradecimentos}

Agradecimento em especial aos biólogos Airan Protázio, Arielson Protázio e a Rafaela Moreira pelo conhecimento compartilhado e apoio à realização deste trabalho. Agradecemos também ao Laboratório Tropical de Primatologia e aos seus funcionários por permitirem a realização das atividades nas suas dependências e colaborarem com o transcorrer das atividades. Aos revisores anônimos pela avaliação crítica do manuscrito.

\section{Referências}

Almeida A.M.R., Margarido T.C.C. \& Filho E.L.D.A.M. (2008) Influência do enriquecimento ambiental no comportamento de primatas do gênero Ateles em Cativeiro. Arquivos de Ciências Veterinárias e Zoologia da Unipar, 11(2): 97-102.

Altmann J. (1974) Observational study of behavior: sampling methods. Behaviour, 49(1): 227267.

Ayres M., Ayres M.J., Ayres D.L. \& Santos S.A. (2007) Bioestat 5.0: aplicações estatísticas nas áreas das Ciências Biomédicas. Belém: Mamirauá/CNPq. 384 p.

Bloomsmith M.A., Brent L.Y. \& Schapiro S.J. (1991) Guidelines for developing and managing an environmental enrichment program for nonhuman Primates. Laboratory Animal Science, 41(4): 372-377.

Boere V. (2001) Environmental enrichment for neotropical primates in captivity. Ciência Rural, Santa Maria, 31(3): 543-551.

Borges M.P., Byk J. \& Del-claro K. (2011) Influência de técnicas de enriquecimento ambiental no aumento do bem-estar de Callithrix penicillata (E. Geoffroy, 1812) (Primates: Callitrichidae). Biotemas, 24(1): 83-94.

Box H.O. (2007) Bem-estar animal (p. 249-270). In: Yamamoto M.E. \& Volpato G.L. (Orgs). Comportamento Animal. Natal: EDUFRN. 520 p.

Buchanan-smith H.M. (2010) Environmental enrichment for neotropical primates in laboratories. Advances in Science \& Research, 5: 41-56.

Carlstead K. \& Shepherdson D. (1994) Effects of environmental enrichment on reproduction. Zoo Biology, 13(5): 447-458.

Castillioni K.P. \& Andrade M.M.M. (2009) Padrão temporal do comportamento do mico-leãopreto (L. chrysopygus) em cativeiro avaliado antes e após a introdução de técnicas de enriquecimento ambiental: um estudo de caso. Monografia, Instituto de Biologia. Universidade Estadual Paulista, São Paulo.

Chamove A., Hosey G. \& Schaetzel P. (1988) Visitors excite primates in zoos. Zoo Biology, 7: 35969.

Gregório D.R. \& Andrade M.M.M. (2010) Etograma de um grupo de micos-leões-da-cara-dourada, Leontopithecus chrysomelas (Kuhl, 1820) (Primates, Callitrichidae) em Cativeiro. Monografia, Instituto de Biologia. Universidade Estadual Paulista, São Paulo.

Guidorizzi C.E. (2008) Ecologia e comportamento do mico-leão-da-cara-dourada, Leontopithecus chrysomelas (Kuhl, 1820) (Primates, Callitrichidae), em um fragmento de floresta semidecidual em Itororó, Bahia, Brasil. Dissertação de Mestrado, Programa de Pós-graduação em Zoologia. Universidade Estadual de Vera Cruz, Ilhéus.

Kierulff M.C.M, Raboy B.E., Procópio-de-oliveira P., Miller K., Passos F.C. \& Prado F. (2008) Ecologia comportamental dos micos-leões (p. 216-250). In: Kleiman D.G. \& Rylands A.B. (Eds). Micos leões: biologia e conservação. Brasília: MMA. 568 p.

Pizzutto C.S., Sgai M.G.F.G. \& Guimarães M.A.B.V. (2009) O enriquecimento ambiental como ferramenta para melhorar a reprodução e o bem-estar de animais cativos. Revista Brasileira de Reprodução Animal, 33(3): 129-138. 
Raboy B.E. \& Dietz J.M. (2004) Diet, foraging, and use of space in wild golden-headed lion tamarins. American Journal of Primatology, 63(1): 1-15.

Reis P.R.P. (2012) Comportamento do mico-leão-da-cara-dourada, Leontopithecus chrysomelas, (Kuhl, 1820) (Primates, Callithrichidae), em áreas de cabruca do sudeste da Bahia. Dissertação de Mestrado, Programa de Pós-graduação em Zoologia. Universidade Estadual de Vera Cruz, Ilhéus.

Ruivo E.B. (2010) EAZA husbandry guidelines for Callitrichidae. $2^{\circ}$ edition. Saint Aignan: Beauval Zoo. $218 \mathrm{p}$. 